\title{
Atrial flutter: prenatal diagnosis and successful neonatal result
}

\author{
Adriana Nieto-Sanjuanero, Roberto Hernández-Monroy*, Mónica M. Briones- Castellanos, \\ Flavio Hernández-Castro, and Ana C. Flores-Domínguez \\ "Dr. José Eleuterio González" University Hospital, Universidad Autónoma de Nuevo León, Monterrey, Nuevo Leon, Mexico
}

\begin{abstract}
Objective: The objective of the study was to present a rare clinical case in neonatology, and emphasize the importance of teamwork that involves its clinical care. Effective communication and timely intervention increase patient success and survival rates. Case: Male patient at 37.4 weeks of gestation with a medical record of a mother with a positive rapid HIV test that during her follow-up appointment presented a persistent fetal heart rate above 220 beats/min, with a report of probable atrial fibrillation and restrictive ductus arteriosus. Programming an emergency cesarean section, a newborn was obtained who received electrical cardioversion, presenting a remission of symptoms. Discussion: A timely detection of prenatal atrial fibrillation was made thanks to the perinatologist, and following the recommendations of the literature, electrical cardioversion was performed. If it had not been detected in a timely manner, the patient could have died. Conclusions: It is significant to highlight that the detection of pathologies prenatally is becoming increasingly more important, since many of them require early and multidisciplinary management. It is fundamental, both for its diagnosis and for the success of the treatment, that the entire medical team is aware of the suspected diagnosis, as well as the immediate post-natal confirmation.
\end{abstract}

Key words: Fibrillation. Atrial. Prenatal. Arrhythmia. Neonatal.

\section{Introduction}

Tachyarrhythmias present an incidence of approximately 1 in every 3000 newborns, of which, $70 \%$ are supraventricular tachycardias and $30 \%$ are atrial flutters $(\mathrm{AFs})^{1}$. AFs constitute $1-2 \%$ of fetal arrhythmias broadly speaking and are generally of an idiopathic origin. Intervention on the fetus will depend on gestational age, hemodynamic repercussions, and linked cardiopathy ${ }^{2}$. This article shows that a multidisciplinary team of well-coordinated specialists is able to detect pathologies prenatally and provides timely and successful treatment with proper communication. In addition, a revision of the literature will be conducted comparing the actions performed in this case.
AF is a supraventricular tachyarrhythmia characterized by rapid atrial activation, between 400 and 700 cycles/ min. It is disorganized, with the consequent impairment of the atrial mechanical function, and characterized by an atrial/ventricular frequency ratio of 2:1 or greater, which causes heart failure, fetal hydrops, and death. There is a hypothesis that diastolic dysfunction, with left atrial enlargement, may predispose babies of diabetic mothers to atrial arrythmias ${ }^{3}$.

In general, around $80 \%$ of patients are asymptomatic; however, $20 \%$ of cases present heart failure with tachypnea and difficulty swallowing, or data of cardiogenic shock ${ }^{4}$.

AF diagnosis by electrocardiogram is simple with sawtooth flutter waves, which are better seen in leads

\section{Correspondence:}

*Roberto Hernández-Monroy

E-mail: robe.23@ hotmail.com
Available online: $30-10-2020$

Date of reception: 05-02-2020

Date of acceptance: $28-04-2020$ DOI: 10.24875/RMU.20000033
Medicina Universitaria. 2020;22(3):127-130 www.medicinauniversitaria.org (http://creativecommons.org/licenses/by-nc-nd/4.0/). 
II, III, and aVF. In absence of structural cardiopathy, atrial fibrillation is observed almost exclusively in babies during their third trimester or at birth ${ }^{5}$.

Its detection forces us to rule out:

1. Ebstein's anomaly.

2. Transposition of great vessels.

3. Wolff-Parkinson-White syndrome.

4. Intra-atrial catheter stimulation.

5. Non-immune fetal hydrops.

It may present as an isolated AF (single episode, and isolated), or recurrent (two or more episodes of $\mathrm{AF}$ ).

When ventricular frequency increases, some of the bundles in the branches may become blocked and produce an aberrated QRS complex, usually of the right branch.

One of the most severe complications is the formations of thrombi, mainly in the left appendage and its link to peripheral embolism, especially to the central nervous system ${ }^{6}$. When the atrial response is very quick, ventricular fibrillation and sudden death may occur. In fetuses with associated edema, linked with arrythmia, mortality may increase from $15 \%$ to $40 \%$, compared to $0 \%$ to $4 \%$ in fetuses without hydrops 7 .

\section{Treatment}

Electric cardioversion as first measure: $0.5-1 \mathrm{~J} / \mathrm{Kg}$ (success in $87 \%$ ).

Prenatal: pharmacologic digital cardioversion (0.25-0.5 mg orally, 3 times a day), flecainide $(10-150 \mathrm{mg}$ orally twice a day), sotalol ( $80 \mathrm{mg}$ every $12 \mathrm{~h}$ ), and in case of term pregnancies, the possibility of interruption of the pregnancy ought to be evaluated ${ }^{1}$.

Other antiarrhythmics, including propranolol, amiodarone, procainamide, or verapamil, are also useful.

The most profitable method of cardioversion in a newborn with AF to date is electrical cardioversion, which also has the highest success rate ${ }^{8}$. Current energy recommendations for synchronized cardioversion of stable supraventricular tachycardia in children is $0.5-1 \mathrm{~J} / \mathrm{kg}$ for the first attempt and 1-2 J/kg for subsequent attempts if the first is not successful. Some of the factors that may impact the success of electrical cardioversion treatment in adults are those which increase thoracic impedance, such as: pericardial effusion, the use of medications such as amiodarone and intubation at the moment of cardioversion. However, it has not been demonstrated that these factors have an impact on neonatal patients ${ }^{3}$.

Regarding prenatal management, digoxin is the treatment of choice with a success rate of $45.1 \%$. It is also possible to use amiodarone and flecainide ${ }^{9}$. Recent studies have shown the effectiveness of digoxin-based prenatal treatments as a first line of management with a success rate of $59 \%$, increasing to $93 \%$ when linked to another antiarrhythmic such as sotalol (included in first line protocols) and flecainide as part of a second line of treatment but only in patients without associated hydrops. There are studies that recommend flecainide as a first line pharmaceutical for the treatment of fetuses with hydrops, yet the majority of the samples of these studies were patients with supraventricular tachycardia ${ }^{10-12}$. This is possible since the bioavailability of flecainide is superior, since it passes through the placental barrier and reaches therapeutic levels in the blood of the umbilical cord even in hydrops fetalis, while digoxin in monotherapy is lower due to the alteration of placental transfer ${ }^{13}$.

Adverse maternal events linked to antiarrhythmics include gastrointestinal symptoms in addition to rhythm alterations which may effect of up to $78 \%$ of patients, being more frequent in cases that are managed with combined therapies. However, most of these adverse events are mild, and a reduction of the dosage allows the continuation of treatment.

We ought to consider the presence of fetal hydrops or the lack thereof for prenatal success, since we have seen that the level of digoxin in serum in hydroponic fetuses is between $20 \%$ and $50 \%$ of the levels of maternal serum, in contrast to the patients without hydrops, who present $80 \%$ and $100 \%{ }^{10}$. Resolution of tachyarrhythmias managed prenatally can delayed in non-hydrops fetuses, from a 3-day median, and with the presence of edema, up to 12.5 days, considering that a second pharmaceutical may be necessary for the resolution of the clinical picture ${ }^{14}$.

Concerning recurrences, they may reappear in the following 2 weeks, even if it is well-controlled when managed prenatally ${ }^{8}$. Maintenance of treatment postnatally will not be necessary when the flutter reverts to sinus rhythm spontaneously or easily responds to electrical conversion. Recurrences generally occur in the first $24 \mathrm{~h}$ of the initial episode, in patients with associated arrhythmias and those who have linked structural anomalies. These patients usually receive beta-blockers or digoxin and beta-blockers over 6-12 months, even though recurrences are usually rare ${ }^{4,15}$.

\section{Clinical case presentation}

The patient, a 25-year-old female, first pregnancy, was found to be HIV+ during pregnancy, treated with tenofovir, emtricitabine, and raltegravir, with an undetectable viral load and CD4 $<500$. An ultrasound was reported by fetal 
medicine: single fetus, alive, cephalic, with fetal tachycardia, biparietal diameter $90.1 \mathrm{~mm}, 36.3$ weeks of gestation (WOG), cephalic circumference $327.5 \mathrm{~mm}, 37.1 \mathrm{WOG}$, abdominal circumference $336.9 \mathrm{~mm}, 37.4$ WOG, femoral longitude $67.9 \mathrm{~mm}, 34.6 \mathrm{WOG}$, humeral longitude $59 \mathrm{~mm}$, 34.1 WOG, average fetometry 36 WOG, estimated fetal weight of $3029 \mathrm{~g}$ (89.6 percentile), amniotic fluid index 3 $\mathrm{cm}$ (oligohydramnios), fetal tachycardia $>200$ beats $/ \mathrm{min}$ (bpm) (FCF $238-587$ bpm), atrial rate (474 bpm)/ventricular frequency (238 bpm)/2:1, abnormal myocardial contractibility, non-pulsatile umbilical vein, wave $A$ ductus venous, AF diagnosed, and restrictive ductus arteriosus.

Given these findings, pregnancy interruption through abdominal c-section was decided on, obtaining a masculine fetus, 37.4 WOG/Capurro, weight $3080 \mathrm{gr}$, Apgar 879, heart rate (HR) $230 \mathrm{bpm}$, and palpable peripheral pulses. He is taken to the neonatal intensive care unit for monitoring, omphaloceles, and a solution plan, with a HR of $230 \mathrm{bpm}$, mean arterial tension of $50 \mathrm{mmHg}, \mathrm{SaO}_{2}$ $99 \%$, and a respiratory frequency of 42 , thoracoabdominal X-ray normal (Fig. 1). An electrocardiogram confirms $\mathrm{AF}$, and an echocardiogram conducted by a pediatric cardiologist reported a structurally normal heart, corroborating the AF diagnosis (Fig. 2). We intubated preceding sedoanalgesia with fentanyl $(0.5 \mathrm{mcg} / \mathrm{kg} / \mathrm{do}$ single dose) and midazolam (100 mcg/kg/dose), and an electrical cardioversion was performed at 1.5 joules obtaining immediate sinus rhythm (Fig. 3). We continued with amiodarone (7 mcg $/ \mathrm{kg} / \mathrm{min}$ in infusion) for $48 \mathrm{~h}$ and later with propranolol at $0.5 \mathrm{mg} / \mathrm{kg}$ every $8 \mathrm{~h}$.

With a favorable evolution, extubation was accomplished at $48 \mathrm{~h}$. We monitored for $72 \mathrm{~h}$ more, continuing with sinus rhythm and feeding adequately egressing with propranolol, zidovudine, and lamivudine and with follow-up appointments at neonatology, cardiology and infectiology.

\section{Discussion}

The present case is an infrequent case in neonatology, one which stood out because of its timely intervention by the perinatology, neonatology, and cardiology teams.

In this case, a well-timed detection of a prenatal atrial fibrillation was performed thanks to a control obstetric ultrasound. With the early diagnosis, we opted for the interruption of pregnancy through an emergency C-section since the patient was at term and, as previously mentioned, success rates were higher in post-natal management with electrical cardioversion than with pharmacological prenatal management, notifying the medical team of neonatology and pediatric cardiology.

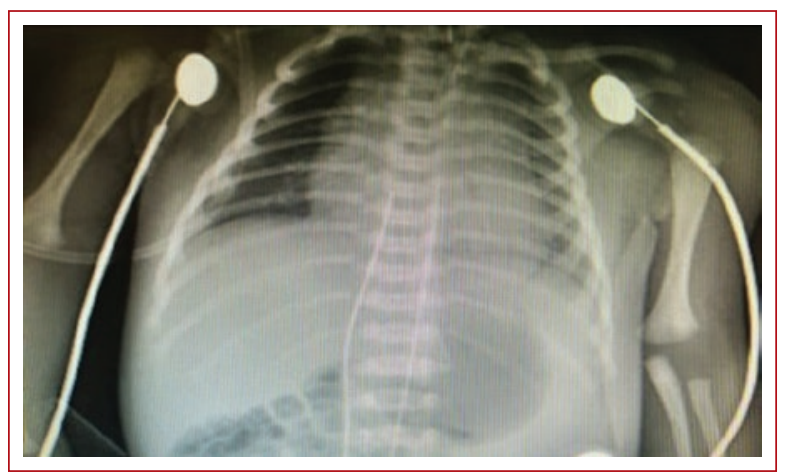

Figure 1. Admission $X$-ray: plain anteroposterior thoracoabdominal $X$-ray showing both lung fields with normal appearances, with a well-defined cardiac silhouette, and other findings, both arterial and venous omphaloskepsis catheters, shown in adequate positions.

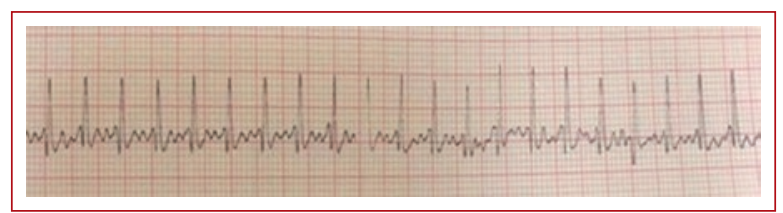

Figure 2. Electrocardiogram performed before electrical cardioversion showing the characteristic "sawtooth" pattern corresponding to atrial fibrillation; the heart rate is $>240$ beats $/ \mathrm{min}$.

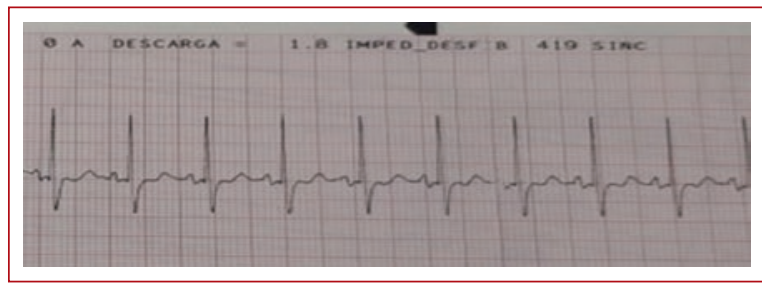

Figure 3. Electrocardiogram after electrical cardioversion: this electrocardiogram shows a sinus rhythm with an estimated heart rate of 140 beats per minute, the complex "qrs is normal."

Taking into consideration the fact that the patient could have suffered rhythm changes during his birth transition and for medical-legal purposes, post-natal diagnostic confirmation was necessary by the previously mentioned team of physicians.

The management, once confirmed, consisted of electrical cardioversion as described in the bibliography, keeping in mind the possible complications and anticipating the possibility of cardiac rhythm change to 
ventricular fibrillation, supraventricular tachycardia, and cardiac arrest was justified by prior authorization from the father to sedate and intubate the patient, securing airways, and monitoring vital signs before treatment, which resulted a success without presenting failure nor recurrence of the AF.

Hospital stay was extended discretely since the withdrawal of sedation was gradual, subsequently beginning the period of highest risk of recurrence, leading to monitoring, and full echocardiographic assessment to rule out structural anomalies.

Without the timely detection of atrial fibrillation, perhaps the patient would have had passed away prenatally without even a certain diagnosis. As mentioned before, the risk of recurrence is low, and even though some articles mention that prophylactic management may not be necessary, we decided to leave management with antiarrhythmics during hospital monitoring and subsequently with propranolol, with successive appointments with cardiology to consider whether or not management would continue for 6 or 12 months as mentioned in the bibliography.

\section{Conclusions}

Prenatal care by the perinatologist alongside the neonatologist has become increasingly important, since timely perinatal diagnosis of different pathologies can provide time to perform ample guidance and family support. Similarly, it has been proven that neonatal mortality is affected when there is a possibility to plan a better therapeutic option and with enough time to put together a multidisciplinary team necessary for proper care.

In the same way, it is essential to stress the importance of continuous monitoring during pregnancy and prenatal control by the perinatologist, since they are the most adequate physician for this task, taking into account the fact that he/she has the best training for the detection of pathologies, and structural as well as fetal heart rhythm, just as in the presented case.

The success of the present case was thanks to timely detection and proper treatment by a multidisciplinary team. It is important not to forget the follow-up after the patient discharge.

\section{Conflicts of interest}

The authors declare that they not have any conflicts of interest.

\section{Financing}

The authors declare that this project did not require any financing.

\section{Ethical disclosures}

Protection of human and animal subjects. The authors declare that the procedures followed were in accordance with the regulations of the relevant clinical research ethics committee and with those of the code of ethics of the world medical association (Declaration of Helsinki).

Confidentiality of data. The authors declare that they have followed the protocols of their work center on the publication of patient data.

Right to privacy and informed consent. The authors have obtained the written informed consent of the patients or subjects mentioned in the article. The corresponding author is in possession of this document.

\section{References}

1. Muñoz H, Copado Y, Díaz C, Muñoz G, Enríquez G, Aguilera S. Diagnóstico y manejo prenatal de patología cardíaca fetal. Rev Med Clin Condes. 2016;27:447-75.

2. Inzaurralde I, Pose G. Hidrops fetal por flutter auricular: a propósito de un caso. Arch Pediatr Urug. 2015;86:304-8.

3. Ceresnak SR, Starc TJ, Hordof AJ, Pass RH, Bonney WJ, Liberman L. Elevated impedance during cardioversion in neonates with atrial flutter. Pediatr Cardiol. 2009;30:436-40.

4. Kaltenbach G, Pérez S, Vallejo C. Aleteo auricular neonatal. Arch Argent Pediatr. 2007;105:427-35.

5. Jaeggi E, Öhman A. Fetal and neonatal arrhythmias. Clin Perinatol. 2016:43:99-112.

6. Wren C. Cardiac arrhythmias in the fetus and newborn. Semin Fetal Neonatal Med. 2006;11:182-90

7. Miyoshi T, Maeno Y, Hamasaki T, Inamura N, Yasukochi S, Kawataki M, et al. Antenatal therapy for fetal supraventricular tachyarrhythmias: multicenter trial. J Am Coll Cardiol. 2019;74:874-85.

8. Prasad D, Steinberg J, Snyder C. Cost-effectiveness of digoxin, pacing, and direct current cardioversion for conversion of atrial flutter in neonates. Cardiol Young. 2018;28:725-9.

9. Garrido-García LM, Delgado-Onofre MG. Trastornos del ritmo en el recién nacido. Acta Pediatr Mex. 2014;35:148-58.

10. Sridharan S, Sullivan I, Tomek V, Wolfenden J, Škovránek J, Yates R, et al. Flecainide versus digoxin for fetal supraventricular tachycardia: comparison of two drug treatment protocols. Heart Rhythm. 2016;13:1913-9.

11. Alsaied T, Baskar S, Fares M, Alahdab F, Czosek RJ, Murad MH, et al. First-line antiarrhythmic transplacental treatment for fetal tachyarrhythmia: a systematic review and meta-analysis. J Am Heart Assoc. 2017;6:e007164.

12. Vergani P, Mariani E, Ciriello E, Locatelli A, Strobelt N, Galli M, et al. Fetal arrhythmias: natural history and management. Ultrasound Med Biol. 2005;31:1-6.

13. Ekiz A, Kaya B, Bornaun H, Acar DK, Avci ME, Bestel A, et al. Flecainide as first-line treatment for fetal supraventricular tachycardia. J Matern Fetal Neonatal Med. 2018;31:407-12.

14. van Engelen A, Weijtens O, Brenner JI, Kleinman CS, Copel JA, Stoutenbeek $P$, et al. Management outcome and follow-up of fetal tachycardia. J Am Coll Cardiol. 1994;24:1371-5.

15. Lisowski L, Verheijen P, Benatar AA, Soyeur DJ, Stoutenbeek P, Brenner Jl, et al. Atrial flutter in the perinatal age group: diagnosis, management and outcome. J Am Coll Cardiol. 2000;35:771-7. 